\title{
Application of extracorporeal cardiopulmonary resuscitation in adult patients with refractory cardiac arrest
}

\author{
Ji-Ge Guo, Jie Cao, Wei-Min Zhang, Fan-Gang Meng, Zheng Zhang, Bi-Jun Xu, Xi-Ming Qian \\ Department of Cardiac Surgery, Sir Run Run Shaw Hospital, College of Medicine, Zhejiang University, Hangzhou, China \\ Contributions: (I) Conception and design: JG Guo, XM Qian; (II) Administrative support: XM Qian; (III) Provision of study materials or patients: \\ J Cao, WM Zhang, FG Meng, Z Zhang, BJ Xu; (IV) Collection and assembly of data: J Cao, WM Zhang, FG Meng, Z Zhang, BJ Xu; (V) Data \\ analysis and interpretation: JG Guo, XM Qian; (VI) Manuscript writing: All authors; (VII) Final approval of manuscript: All authors. \\ Correspondence to: Xi-Ming Qian, MD. Department of Cardiac Surgery, Sir Run Run Shaw Hospital, College of Medicine, Zhejiang University, No.3 \\ Qingchundong Road, Hangzhou 310016, China. Email: gjgcpb1278a@163.com.
}

\begin{abstract}
Background: The aim of this study was to summarize the clinical experience of extracorporeal cardiopulmonary resuscitation (ECPR) in the treatment of adult patients with refractory cardiac arrest.

Methods: The clinical data of 12 cases of adult patients with cardiac arrest hospitalized between June 2015 and September 2019 who were unable to achieve return of spontaneous circulation effectively with conventional cardiopulmonary resuscitation (CCPR) and were treated with ECPR technology were retrospectively analyzed. The group included six males and six females aged between 18 and 69 years. All the patients underwent veno-arterial extracorporeal membrane oxygenation (V-A ECMO) support with the adoption of femoral artery and vein catheterization.

Results: The duration of cardiopulmonary resuscitation (CPR) for the 12 patients was 32-125 min, and the ECMO duration was 2-190 h. Four patients were successfully weaned from ECMO and survived until hospital discharge. The other eight patients died in hospital; hemodynamic collapse (four patients) in the early stage of ECMO and severe neurological complications (three patients) were the main causes of death.

Conclusions: Single-center data showed that ECPR provided a new rescue alternative for some patients with reversible refractory cardiac arrest. We have demonstrated that the success rate of treatment could be improved by selecting suitable patients and reducing the CPR duration as much as possible.
\end{abstract}

Keywords: Cardiac arrest; cardiopulmonary resuscitation (CPR); extracorporeal membrane oxygenation (ECMO)

Submitted Apr 03, 2020. Accepted for publication Dec 03, 2020.

doi: $10.21037 /$ jtd-20-1573

View this article at: http://dx.doi.org/10.21037/jtd-20-1573

\section{Introduction}

Cardiac arrest caused by a variety of factors has always been a serious, life-threatening emergency state. Conventional cardiopulmonary resuscitation (CCPR) is the basic method used to treat cardiac arrest; however, the success rate of rescue has not been high (1-3). With the extension of cardiopulmonary resuscitation (CPR) duration, the probability of achieving return of spontaneous circulation (ROSC) is increasingly small. How to improve the rescue success rate of patients with cardiac arrest has been a focus of medical research. In recent years, with the development of extracorporeal life support technology, extracorporeal cardiopulmonary resuscitation (ECPR) derived from extracorporeal membrane oxygenation (ECMO) has been increasingly applied to patients with CCPR failure. It has been reported that ECPR could improve the survival rate and the neurological prognosis of patients with cardiac arrest (4-6).

The aim of this study was to retrospectively analyze the clinical data of patients with cardiac arrest who were treated with ECPR at Sir Run Run Shaw Hospital in recent years to summarize the experience of the treatment of patients with cardiac arrest. We present the following article in accordance with the STROBE checklist (available at http:// dx.doi.org/10.21037/jtd-20-1573). 


\section{Methods}

This study included 12 adult patients with cardiac arrest who underwent ECPR at our hospital between June 2015 and September 2019. There were six males and six females aged 18-69 years (average $47.4 \pm 16.3$ years) with body mass of $39-71 \mathrm{~kg}$ (average $61.7 \pm 11.8 \mathrm{~kg}$ ). The inclusion criteria of ECPR patients in our center were that they did not achieve ROSC with CPR for at least $15 \mathrm{~min}$, and they did not have any advanced malignant tumor, irreversible organ dysfunction, or serious nervous system injuries before the onset of the disease. The family members of the patients were informed and agreed to ECMO rescue. Patients with acute heart failure or failed weaning from cardiopulmonary bypass after cardiac surgery who were treated with ECMO but did not undergo CPR were not included in this study.

The study was conducted in accordance with the Declaration of Helsinki (as revised in 2013). The study was approved by institutional ethics board of Sir Run Run Shaw Hospital (2020-578-01) and individual consent for this retrospective analysis was waived.

All the patients underwent veno-arterial (V-A) ECMO support with the adoption of femoral artery and vein catheterization. Ten patients were treated by using skin incision, and two patients were treated by using the percutaneous technique. The ECMO circuit (BE-PLS 2050, Maquet, Germany) was primed with lactate Ringer's solution. After initiation of ECMO, the hematocrit was maintained at $>24 \%$, platelet $>5 \times 10^{9} / \mathrm{L}$, fibrinogen $>1.0 \mathrm{~g} / \mathrm{L}$. In the early stage of ECMO, echocardiography was fully used to assess the cardiac function and filling extent to avoid left ventricular hyperinflation. According to the recovery of heart rhythm and the state of cardiac function, the perfusion flow rate was adjusted appropriately. Combined with the use of vasoactive drugs, the mean arterial pressure should be maintained above $65 \mathrm{mmHg}$, and the mixed venous oxygen saturation should be more than $65 \%$. If this was the case, the blood-gas and biochemical index tended to be improved. The mechanical ventilator was set up with a protective lung ventilation strategy: the initial tidal volume was $6-8 \mathrm{~mL} /$ $\mathrm{kg}$, positive end-expiratory pressure (PEEP) $5-10 \mathrm{cmH}_{2} \mathrm{O}$, respiratory rate was $10-12$ times $/ \mathrm{min}, \mathrm{FiO}_{2}$ was $40-50 \%$, and distal limb perfusion of the intubated side was performed routinely. The anticoagulation strategy adopted heparin titrated to maintain activated clotting time (ACT) for 160$200 \mathrm{~s}$ and activated partial thromboplastin time (APTT) for 50-80 s. If the groin incision still had obvious bleeding after complete surgical hemostasis or increased drainage from the chest tube, the requirements of anticoagulation were properly reduced, or could be suspended for a short time. In the early stage of ECPR, the temperature of the patient was controlled at $34-36^{\circ} \mathrm{C}$ by the heater-cooler. The function of the nervous system was evaluated when the vital signs were stable. If necessary, a brain computerized tomography (CT) examination should be performed to confirm the diagnosis. If the hemodynamic indexes of the patients were significantly improved after several days of ECMO support with no or a small dose of vasoactive drugs and the echocardiography indicated that the myocardial contraction was satisfactory, the ECMO flow rate could be reduced gradually until ECMO was withdrawn.

ECPR technology has only appeared and been developed in China in recent years. It was used for the first time in our hospital in June 2015, and there were 12 cases in total by September 2019. This study included all the patients who were treated with ECPR in our hospital. Data were obtained from our hospital's electronic medical records.

\section{Statistical analysis}

In the present study, the data were statistically analyzed using SPSS statistical software (version 19.0, IBM, USA). The measurement data were expressed as mean \pm standard deviation (SD). Enumeration data were expressed in percentage $(\%)$.

\section{Results}

Twelve patients treated with ECPR were included in this study (Figure 1). It was indicated that the CPR duration of the 12 patients was $32-125 \mathrm{~min}$ (average $71.6 \pm 32.7 \mathrm{~min}$ ), and the ECMO support time was 2-190 hours (average $94.9 \pm 63.2 \mathrm{~min}$ ) (Table 1). Eight patients were successfully weaned from ECMO and four of these patients survived; the survival rate was $33.3 \%$ [4/12]. Among the eight deceased patients, four patients who could not be weaned from ECMO died due to hemodynamic collapse during the early stage of the ECMO treatment. Four patients died after weaning from ECMO; among these, three patients died due to severe neurological complications and one patient died due to recurrent cardiac arrest.

Among the four patients who survived and were discharged from the hospital, one patient suffered from compartment syndrome in the intubated side of the crus, which resulted in partial necrosis of skin and soft tissue. Considering the insufficiency of blood supply at the distal 


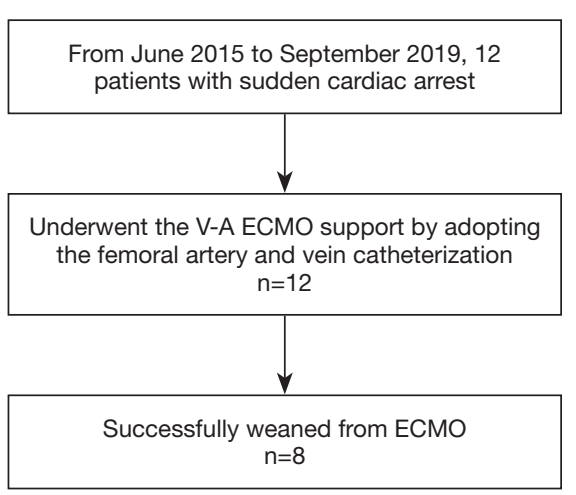

Figure 1 Study flowchart. V-A ECMO, veno-arterial extracorporeal membrane oxygenation.

limb (no additional distal perfusion was established), the crus was preserved after multiple debridement, skin grafting, and other remedial treatment. However, it still resulted in the claudication of the right lower extremity. It was strongly suspected that the cardiac arrest of one patient was caused by a pulmonary embolism. Therefore, the patient underwent urgent thrombolysis with Alteplase before receiving ECMO. During ECMO, the patient experienced gastrointestinal bleeding and abdominal bleeding successively, and an exploratory laparotomy was performed to achieve hemostasis. In addition, the CPR duration of this patient was long $(120 \mathrm{~min})$, and the recovery of organs, including the nervous system, was slow. This patient recovered cardiac function 1 year after discharge. The echocardiography indicated that the value of left ventricular ejection fraction (LVEF) was 0.68 , but the neural responses were sluggish. The follow-up of the other patients ranged from 3 months to 4 years, and the cardiac and nervous system functions of these patients recovered well.

\section{Discussion}

According to the American Heart Association's Get With The Guidelines ${ }^{\circledR}$-Resuscitation (GWTG-R), among 84,625 patients with cardiac arrest hospitalized between 2000 and 2009, the survival-to-discharge rate ranged from $13.7 \%$ to $22.3 \%$ (1). Although there is no large-scale randomized controlled clinical study to confirm the effect of ECPR, according to a large number of retrospective studies and meta-analyses conducted in the past, most scholars believe that ECPR can benefit specific populations $(2,6)$. At present, there are different reports on the efficacy of ECPR in the treatment of in-hospital cardiac arrest; however, the survival rate in most studies is between $30 \%$ and $40 \%(7-11)$. The survival rate of our group of patients is $33.3 \%$ (4/12), which is in line with most of the literature reports. Regarding the outcome of refractory cardiac arrest treated by ECPR, it is generally accepted that the duration of low blood flow is one of the most important factors (11-13). The low blood flow duration refers to the period from the start of CPR to ECMO initiation. The shorter the time, the more favorable the prognosis will be. Generally, $60 \mathrm{~min}$ is a watershed, and the recovery probability will decrease if $60 \mathrm{~min}$ is exceeded $(14,15)$. Therefore, it is critical that ECMO is quickly established for patients with cardiac arrest, including the assessment of indications and the rapid response of the ECMO team. At our center, the duration from cardiac arrest to ECMO initiation varies widely, from 32 to $125 \mathrm{~min}$. The main reason is that we have not established a 24-hour duty system and sometimes the personnel and equipment cannot arrive in time. We had six patients with CPR duration of more than $60 \mathrm{~min}$ (five deaths), which directly affected the prognosis of our patients. We were also learning to improve the ECPR rescue process to control the CPR duration within $60 \mathrm{~min}$.

In addition to reducing the CPR duration as much as possible, the quality of CPR is essential (16). It has been reported that chest compression can provide about $25 \%$ of cardiac output (17). High-quality compression means that important organs such as the heart and brain can obtain more blood perfusion. A patient with cardiac arrest in our hospital survived even though the CPR duration was $120 \mathrm{~min}$. The patient might have benefited from highquality CPR. It is suggested that for some special patients, although the CPR duration has exceeded the recognized range, it has the significance of active rescue, especially for young patients whose blood pressure can be maintained or in whom vital signs appear during CPR, such as wheezing, pupillary reflex, and limb movement.

Femoral artery and vein cannulation were applied to all the patients, and groin area skin incision was used to expose vessels to cannulate by using Seldinger technique in most of the patients. For some patients with better conditions, such as palpable femoral artery pulsation, accessible ultrasound equipment, and experienced percutaneous operators, the percutaneous method (two patients) could be used. It is not clear whether cannulation or the percutaneous method is better for the incision. We believe it is essential that the CPR duration should be reduced as much as possible. Regarding the selection of cannula size, we learned a lesson from our first ECPR patient. Due to the lack of experience, 


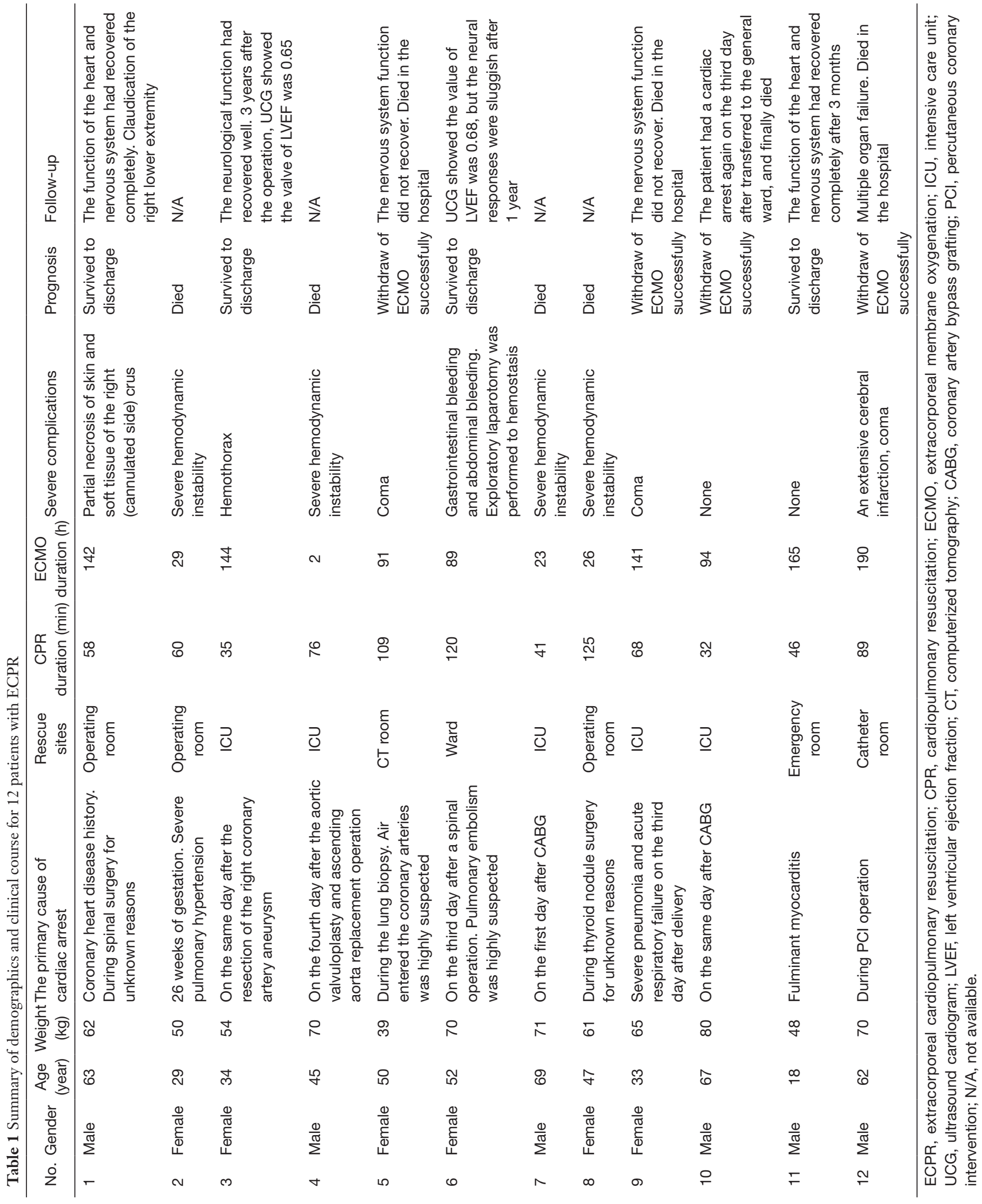


the size of the cannula in the femoral artery was a little large (Edwards $20 \mathrm{Fr}$ ), and no additional distal perfusion was established. Consequently, the blood supply of the distal limb on the intubated side was insufficient, and the skin and soft tissue of the calves were partially necrotic. Therefore, it could be concluded that 16 or $18 \mathrm{Fr}$ is more suitable for femoral artery cannulation; however, 22 or $24 \mathrm{Fr}$ was used for femoral vein cannulation in most of the patients. Furthermore, the additional distal perfusion was implemented routinely. The method is to place the vessel sheath of $8 \mathrm{Fr}$ in the distal direction of the superficial femoral artery in the same incision and connect it with the main femoral cannula. Moreover, the skin temperature, color and circumference of the intubated side should be observed regularly, and the blood flow of the lower extremity should be monitored by ultrasound if necessary. After the improvement of the above measures, the problem of insufficient blood supply to the limbs on the intubated side was resolved.

It has been reported that the initial shockable rhythm at the first onset $(17,18)$, the lactate level $(11,16,19)$, and the partial pressure of end-tidal carbon dioxide before ECPR (20) can be regarded as prognostic indicators in patients with cardiac arrest. Some data were not collected completely and could not be further analyzed; however, the lactate level was recorded in detail immediately when running ECPR. The lactate level at the point of initiation of ECPR of the four surviving patients and eight deceased patients was $12.1 \pm 2.0$ and $15.2 \pm 4.1 \mathrm{mmol} / \mathrm{L}$, respectively. It could be seen that the level of blood lactate in the survival group was lower than that in the deceased group, which was consistent with the literature. This indicated that patients in the survival group possessed a relatively mild degree of tissue ischemia and hypoxia during CPR, so the prognosis was better, which had a certain reference significance for whether ECPR treatment needed to be started.

It was found that bleeding was a common and serious complication of ECPR. Various degrees of bleeding at the cannula site occurred in these 12 patients. Two patients experienced pulmonary contusion caused by chest compression, with obvious complications such as endotracheal hemorrhage and hemothorax. One patient experienced gastrointestinal hemorrhaging first, then abdominal hemorrhaging. An emergency operation had to be performed, and a rupture of the diaphragm artery was found. Therefore, the timely judgment and treatment of bleeding during ECMO are very important to stabilize the condition.

The limitation of this study is that it was a retrospective analysis with a small number of patients and the lack of a control group for comparison, and therefore the persuasion of the data is relatively limited. It is expected that more in-depth research in this field will be conducted with the increase in the number of patients in the future.

\section{Conclusions}

Single-center data showed that ECPR provided a new rescue alternative for some patients with reversible refractory cardiac arrest. We have demonstrated that the success rate of treatment could be improved by selecting suitable patients and reducing CPR duration as much as possible.

\section{Acknowledgments}

Funding: This study was supported by National Key R\&D Program of China (No. 2017YFC1308000).

\section{Footnote}

Reporting Checklist: The authors have completed the STROBE reporting checklist. Available at http://dx.doi. org/10.21037/jtd-20-1573

Data Sharing Statement: Available at http://dx.doi. org/10.21037/jtd-20-1573

Conflicts of Interest: All authors have completed the ICMJE uniform disclosure form (available at http://dx.doi. org/10.21037/jtd-20-1573). The authors have no conflicts of interest to declare.

Ethical Statement: The authors are accountable for all aspects of the work in ensuring that questions related to the accuracy or integrity of any part of the work are appropriately investigated and resolved. The study was conducted in accordance with the Declaration of Helsinki (as revised in 2013). The study was approved by institutional ethics board of Sir Run Run Shaw Hospital (2020-578-01) and individual consent for this retrospective analysis was waived.

Open Access Statement: This is an Open Access article distributed in accordance with the Creative Commons Attribution-NonCommercial-NoDerivs 4.0 International License (CC BY-NC-ND 4.0), which permits the non- 
commercial replication and distribution of the article with the strict proviso that no changes or edits are made and the original work is properly cited (including links to both the formal publication through the relevant DOI and the license). See: https://creativecommons.org/licenses/by-nc-nd/4.0/.

\section{References}

1. Girotra S, Nallamothu BK, Spertus JA, et al; American Heart Association Get with the Guidelines-Resuscitation Investigators. Trends in survival after in-hospital cardiac arrest. N Engl J Med 2012;367:1912-20.

2. Wang GN, Chen XF, Qiao L, et al. Comparison of extracorporeal and conventional cardiopulmonary resuscitation: A meta-analysis of 2260 patients with cardiac arrest. World J Emerg Med 2017;8:5-11.

3. Pozzi M, Armoiry X, Achana F, et al. Extracorporeal Life Support for Refractory Cardiac Arrest: A 10-Year Comparative Analysis. Ann Thorac Surg 2019;107:809-16.

4. Siao FY, Chiu CC, Chiu CW, et al. Managing cardiac arrest with refractory ventricular fibrillation in the emergency department: Conventional cardiopulmonary resuscitation versus extracorporeal cardiopulmonary resuscitation. Resuscitation 2015;92:70-6.

5. Prodhan P, Fiser RT, Dyamenahalli U, et al. Outcomes after extracorporeal cardiopulmonary resuscitation (ECPR) following refractory pediatric cardiac arrest in the intensive care unit. Resuscitation 2009;80:1124-9.

6. Shin TG, Jo IJ, Sim MS, et al. Two-year survival and neurological outcome of in-hospital cardiac arrest patients rescued by extracorporeal cardiopulmonary resuscitation. Int J Cardiol 2013;168:3424-30.

7. Lan C, Tsai PR, Chen YS, et al. Prognostic factors for adult patients receiving extracorporeal membrane oxygenation as mechanical circulatory support--a 14-year experience at a medical center. Artif Organs 2010;34:E59-64.

8. Lin JW, Wang MJ, Yu HY, et al. Comparing the survival between extracorporeal rescue and conventional resuscitation in adult in-hospital cardiac arrests: propensity analysis of three-year data. Resuscitation 2010;81:796-803.

9. Kim SJ, Kim HJ, Lee HY, et al. Comparing extracorporeal cardiopulmonary resuscitation with conventional cardiopulmonary resuscitation: A meta-analysis. Resuscitation 2016;103:106-16.

10. Ahn C, Kim W, Cho Y, et al. Efficacy of extracorporeal cardiopulmonary resuscitation compared to conventional cardiopulmonary resuscitation for adult cardiac arrest patients: a systematic review and meta-analysis. Sci Rep
2016;6:34208.

11. D'Arrigo S, Cacciola S, Dennis M, et al. Predictors of favourable outcome after in-hospital cardiac arrest treated with extracorporeal cardiopulmonary resuscitation: A systematic review and meta-analysis. Resuscitation 2017;121:62-70.

12. Wengenmayer T, Rombach S, Ramshorn F, et al. Influence of low-flow time on survival after extracorporeal cardiopulmonary resuscitation (eCPR). Crit Care 2017;21:157.

13. Singer B, Reynolds JC, Lockey DJ, et al. Pre-hospital extra-corporeal cardiopulmonary resuscitation. Scand J Trauma Resusc Emerg Med 2018;26:21.

14. Kim DH, Kim JB, Jung SH, et al. Extracorporeal Cardiopulmonary Resuscitation: Predictors of Survival. Korean J Thorac Cardiovasc Surg 2016;49:273-9.

15. Michels G, Wengenmayer T, Hagl C, et al. Recommendations for extracorporeal cardiopulmonary resuscitation (eCPR): consensus statement of DGIIN, DGK, DGTHG, DGfK, DGNI, DGAI, DIVI and GRC. Clin Res Cardiol 2019;108:455-64.

16. Wang J, Ma Q, Zhang H, et al. Predictors of survival and neurologic outcome for adults with extracorporeal cardiopulmonary resuscitation: A systemic review and meta-analysis. Medicine (Baltimore) 2018;97:e13257.

17. Lee SW, Han KS, Park JS, et al. Prognostic indicators of survival and survival prediction model following extracorporeal cardiopulmonary resuscitation in patients with sudden refractory cardiac arrest. Ann Intensive Care 2017;7:87.

18. Chen Z, Liu C, Huang J, et al. Clinical Efficacy of Extracorporeal Cardiopulmonary Resuscitation for Adults with Cardiac Arrest: Meta-Analysis with Trial Sequential Analysis. Biomed Res Int 2019;2019:6414673.

19. Jung C, Bueter S, Wernly B, et al. Lactate Clearance Predicts Good Neurological Outcomes in Cardiac Arrest Patients Treated with Extracorporeal Cardiopulmonary Resuscitation. J Clin Med 2019;8:374.

20. Patricio D, Peluso L, Brasseur A, et al. Comparison of extracorporeal and conventional cardiopulmonary resuscitation: a retrospective propensity score matched study. Crit Care 2019;23:27.

Cite this article as: Guo JG, Cao J, Zhang WM, Meng FG, Zhang Z, Xu BJ, Qian XM. Application of extracorporeal cardiopulmonary resuscitation in adult patients with refractory cardiac arrest. J Thorac Dis 2021;13(2):831-836. doi: 10.21037/ jtd-20-1573 